\title{
EFECTO DE LA PODA SOBRE LA CALIDAD DE FRUTOS DE Vitis vinifera L. SAUVIGNON BLANC DURANTE LA MADURACIÓN
}

\section{EFFECT OF PRUNING ON FRUIT QUALITY OF Vitis vinifera $L$. SAUVIGNON BLANC DURING MATURATION}

\author{
Ingrid Y. Walteros ${ }^{1}$, Deisy C. Molanoํ, Pedro J. Almanza-Merchán² \\ Recibido para publicación: Agosto 7 de 2012 - Aceptado para publicación: Noviembre 5 de 2012
}

\begin{abstract}
RESUMEN
La producción y calidad de la uva para elaboración de vino, se modifican con las prácticas culturales que se realizan en el viñedo. La poda es una de las prácticas que buscan mejorar la calidad final de mostos y vinos. Con la investigación se buscó conocer el efecto de tres tipos de poda (corta, larga y mixta) en la evolución de los componentes de la calidad, durante la maduración de frutos de Vitis vinifera L. var. Sauvignon Blanc, en el municipio de Sutamarchán-Boyacá, Colombia. Se recolectaron, cada quince días, aleatoriamente 20 bayas, desde el inicio del envero, hasta la maduración, para determinar el comportamiento de los sólidos solubles totales (SST), acidez total titulable (ATT) e índice de madurez técnica (IMT). Con la poda larga se alcanzó el mayor contenido de azúcares $\left(22,76^{\circ}\right.$ Brix) y el más alto IMT $(3,42)$, la acidez total titulable (ATT) disminuyó con el desarrollo del fruto, obteniéndose en el momento de la vendimia una acumulación de ácidos orgánicos de $6,88 \mathrm{~g} \mathrm{~L}^{-1}$. Los tratamientos no afectaron el contenido de SST; mas si hubo diferencias significativas entre ATT e IMT. La poda larga produjo los frutos con los valores más adecuados, de calidad (SST, ATT, IMT) requeridos para la elaboración de vinos tropicales de la variedad Sauvignon Blanc, en la localidad del estudio.
\end{abstract}

Palabras clave: viticultura de calidad, sólidos solubles, acidez, madurez técnica.

\begin{abstract}
Production and quality of grapes for winemaking change with cultural practices that are made in the vineyard. Pruning is one of the practices that seek to improve the final quality of musts and wines. With the study, aimed to know the effect of three types of pruning (short, long and mixed) on the evolution of quality components during ripening of Vitis vinifera L. var. Sauvignon Blanc in Sutamarchán-Boyacá, Colombia. Every two weeks, since the beginning of veraison to maturity, 20 berries were collected randomly to determine the behavior of

${ }^{1}$ Ingeniera Agrónoma, Facultad de Ciencias Agropecuarias, Universidad Pedagógica y Tecnológica de Colombia Tunja. e-mail: ingridwal89@hotmail.com

${ }^{2}$ Ingeniero Agrónomo, Ph.D. Fisiología de cultivos. Profesor Asociado. Facultad de Ciencias Agropecuarias, Grupo de Investigación Ecofisiología Vegetal. Universidad Pedagógica y Tecnológica de Colombia Tunja. e-mail: ppcalma@gmail.com
\end{abstract}


the total soluble solids (TSS), titratable acidity (TTA) and technical maturity index (TMI). With long pruning the highest sugar content $\left(22.76^{\circ}\right.$ Brix) was reached and higher TMI (3.42), total titratable acidity (TTA) decreased with fruit development, resulting on time of harvest, accumulation of organic acids $\left(6.88 \mathrm{~g} \mathrm{~L}^{-1}\right)$. The long pruning presented the appropriate fruit values, in quality components (TSS, TTA, TMI) for tropical wines of Sauvignon Blanc, in the locality of the study.

Key words: quality viticulture, soluble solids, acidity, technical maturity.

\section{INTRODUCCIÓN}

La producción de uva y su calidad se modifican con las prácticas culturales que el viticultor realiza sobre la planta y el viñedo. Para una situación determinada (variedad, lugar y año), es aceptado que las técnicas que limitan la producción permiten mejorar las cualidades de las uvas y por consiguiente la calidad de los vinos producidos (Sipiora 1995; Togores y Fernández-Cano 2011). Dentro de algunas de las prácticas tendientes a mejorar la calidad final de mostos y vinos, se encuentra el control del vigor de las plantas, las cuales se realizan mediante labores culturales de poda, como por ejemplo, ajustando el número de yemas por metro lineal (Ferreyra et al. 1998; Acevedo et al. 2004).

Según Lavín et al. (2003), la poda de producción se practica durante toda la etapa productiva de la planta y tiene como objetivo principal, asegurar y regular la producción, permitiendo mantener la forma de la planta y su nivel de producción. Esta clase de poda debe adecuarse al vigor de cada planta, y con base en que la producción depende del número de yemas dejadas durante esta operación. Para Smart y Robinson (1991), la intensidad de la poda de producción influye considerablemente sobre la producción de uva, la cantidad de madera como órgano de reserva y sobre la composición de los frutos. Es así, como una mayor cantidad de yemas dejadas en la planta producen una mayor cantidad de pámpanos que generan mayor producción de uvas (Zamboni et al. 1991), pero la calidad de la uva es inferior (Champagnol 1984).

La poda puede ser corta o larga, dependiendo del número de yemas que se dejen en los pitones o pulgares (Almanza et al. 2012). Dentro del tipo de podas cortas, se destacan los sistemas de conducción en vaso y cordón o Royat; y en las podas largas, los de vara o Guyot y sus numerosas variantes (García y Bernabé 1997). De acuerdo con Reynier (1995), es necesario tener cuidado al momento se escoger el tipo de poda que se va a realizar, por ejemplo, al podar dejando un número bajo de yemas, se podría presentar una disminución en la producción de frutos y un aumento del vigor de la planta que generaría frutos de menor calidad; en tanto que, una excesiva carga de yemas conduce a una mayor densidad de brotes, de área foliar y producción; aumentando la relación fuente vertedero, lo que ocasionaría una maduración poco uniforme y una baja calidad de la fruta, además de una lignificación insuficiente y un debilitamiento de la planta (Almanza 2011). Pszczolkowski y Bordeu (1984), afirman que la poda también contribuye con incrementos de acido málico, potasio y $\mathrm{pH}$ de los frutos y a una disminución del color y sólidos solubles totales ( ${ }^{\circ}$ Brix), reduciendo significativamente la calidad del vino. 
'Sauvignon Blanc' es una cepa originaria de Burdeos, Francia, que produce frutos amarilloverdosos con tonalidades doradas al finalizar la maduración, presenta buen potencial de producción y calidad, junto con Chardonnay produce vinos de marcado carácter y potentes aromas primarios (Mapa 2008). Se cultiva en Francia, Chile y California, en Colombia, se siembra en la región del alto Ricaurte en Boyacá (Almanza et al. 2012). Catania y Avagnina (2007) mencionan que se comporta como una variedad vigorosa, de maduración temprana, al momento de la madurez tiene muy buena acidez y cuando madura muy bien, puede llegar a tener una elevada concentración de azúcares. La planta se caracteriza por presentar racimos medianos, compactos y cónicos, el fruto es elipsoidal y de pulpa jugosa y sin pigmentación, con sabor afrutado y muy aromático (Catania y Avagnina 2007).

En la región del alto Ricaurte en Boyacá, aún no se han realizado investigaciones que conduzcan a evaluar el efecto de diferentes tipos de poda sobre el comportamiento de los principales componentes de calidad organoléptica en frutos de uva para elaboración de vinos tropicales (sólidos solubles y acidez), desde el envero hasta la vendimia. Por tanto, el objetivo de la investigación, fue conocer la evolución de los componentes de la calidad durante la maduración de frutos de Vitis vinífera L. var. Sauvignon Blanc bajo tres sistemas de poda en el municipio de Sutamarchán- Boyacá.

\section{MATERIALES Y MÉTODOS}

El trabajo experimental se realizó en el Viñedo Márquez de Villa de Leyva (finca Ain Karin), localizado en el municipio de Sutamarchán,
Boyacá, a los $5^{\circ} 39^{\prime}$ de latitud norte, $73^{\circ}$ $35^{\prime}$ de longitud oeste respecto al meridiano de Greenwich; en una altitud sobre el nivel del mar de 2110 m. La zona se caracteriza por presentar un micro clima apto para la viticultura (Camacho 2012), con alta radiación solar y bajas temperaturas nocturnas. Los suelos son de tipo calcáreo y fertilidad media. La precipitación acumulada fue de 1196,1 $\mathrm{mm}$, con temperatura promedio de $16,9^{\circ} \mathrm{C}$, temperatura máxima absoluta de $25,1^{\circ} \mathrm{C}$ y una media mínima absoluta de $7,6^{\circ} \mathrm{C}$ (IDEAM 2012), durante la investigación se presentó el conocido fenómeno de la niña, caracterizado por altas precipitaciones, siendo esta una situación atípica en la zona.

Las plantas evaluadas correspondieron a la cepa de uva blanca Sauvignon Blanc, injertada sobre el patrón 1103 Paulsen (Vitis rupestris $\mathrm{x}$ Vitis berlandeieri). Las plantas están sembradas con distancia de 1,5 m x $1,0 \mathrm{~m}$. La finca cuenta con un sistema de fertirriego por goteo. Se seleccionaron plantas con características homogéneas de ocho años de edad, en donde se realizaron tres tipos de poda. Para el caso del testigo se evaluó la poda corta (tradicional en el viñedo), dejándose dos yemas en tres sarmientos o pitones; en la poda larga, se dejaron cinco yemas en tres pitones y en la poda mixta, que es el resultado de una combinación de poda larga y poda corta.

Cada quince días, desde el inicio de la maduración del fruto (envero) hasta la cosecha (vendimia), se recolectaron aleatoriamente de cada tipo de poda 20 frutos o bayas, que fueron Ilevadas al laboratorio de fisiología vegetal de la Universidad Pedagógica y Tecnológica de Colombia - Tunja. Se obtuvieron $10 \mathrm{ml}$ de mosto 
y se evaluaron las variables propuestas. Los contenidos de sólidos solubles totales (SST) se determinaron mediante refractómetro manual Atago de 0 a $32 \%$ a $20^{\circ} \mathrm{C}$ y se expresaron en grados Brix ( ${ }^{\circ}$ Brix); la acidez total titulable (ATT), mediante la titulación con $\mathrm{NaOH}$ 0,1 $\mathrm{N}$ hasta $\mathrm{pH} 8,2$ y se expresó como ácido tartárico (AOAC 1990) y el índice de madurez técnico (IMT), se determinó de acuerdo con la metodología propuesta por Parra y Hernández (1997) mediante la relación SST/ATT.

Se utilizó un diseño completamente al azar con tres tratamientos correspondientes a los tipos de poda (corta, larga y mixta), cada tratamiento tuvo cuatro repeticiones, para un total de 12 unidades experimentales, cada unidad experimental estuvo compuesta por dos plantas. Para analizar el efecto de la poda sobre los compuestos de calidad, los datos obtenidos fueron sometidos a un análisis de varianza (Anova) para determinar la presencia de diferencias significativas, se Ilevó a cabo la prueba de Tukey (5\%), con el fin de establecer el mejor tipo de poda. Para el análisis de los datos se utilizó programa estadístico SAS® v. 9.2.

\section{RESULTADOS Y DISCUSIÓN}

\section{Sólidos Solubles Totales}

LOS SST incrementaron continuamente con la maduración de las bayas, durante el envero a los 143 días después de poda (ddp) no se presentaron diferencias significativas con los diferentes tipos de poda. Sin embargo, la poda larga produjo la mayor ganancia de SST $(11,43$ ${ }^{\circ}$ Brix) en tanto que el testigo (poda corta) con $10,9{ }^{\circ}$ Brix y la poda mixta con $10,34{ }^{\circ}$ Brix generó el menor contenido de azúcares, esta tendencia sigue hasta los 175 ddp donde la poda mixta alcanzó los valores más altos de SST con 22,25 ${ }^{\circ}$ Brix, seguido por la poda corta con $21,01^{\circ}$ Brix y con $20,66^{\circ}$ Brix la poda larga (figura 1). Lo anterior queda confirmado por la investigación de Dokoozlian (2000), quien afirma que durante las etapas iníciales de crecimiento de la fruta, el azúcar se encuentra en bajas concentraciones, por lo general alrededor del $2 \%$ del peso de las bayas frescas y a partir de cerca del inicio del envero, la concentración de azúcar aumenta rápidamente, y puede llegar a más del 25\%, en el momento de la cosecha; esto se debe a que los frutos de la uva tienen la capacidad de acumular mayores concentraciones de azúcar durante la fase de maduración (Togores y Fernández-Cano 2011).

Durante el período de maduración y hasta la vendimia (184 ddp) en el fruto de Sauvignon Blanc no se presentaron diferencias significativas en ningún punto de muestreo por el efecto de las podas. Al momento de la vendimia, la poda larga alcanzó el valor más alto $\left(22,76^{\circ} \mathrm{Brix}\right)$ y el menor para la poda corta (testigo) $\left(22,09{ }^{\circ}\right.$ Brix) (Figura 1). Estos resultados establecen la importancia de la mayor área foliar que se presenta en las plantas con poda larga, las cuales generarían

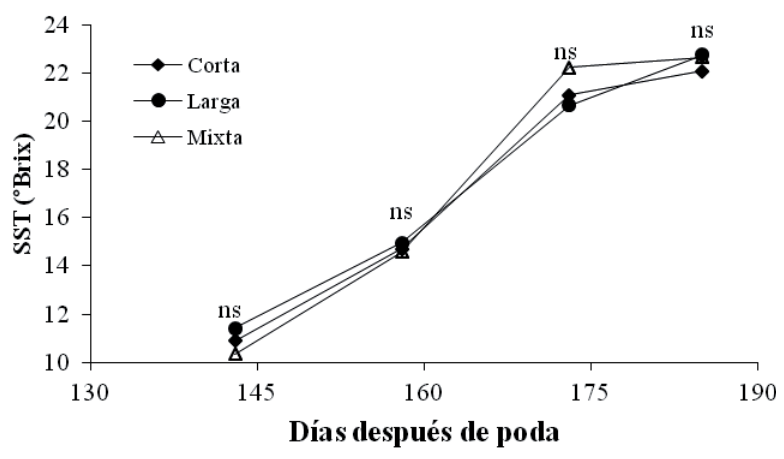

Figura 1. Comportamiento de los Sólidos Solubles Totales (SST) durante la maduración de las bayas de 'Sauvignon Blanc' bajo diferentes tipos de poda. ns: no hay diferencias significativas. 
mayores cantidades de fotosintatos que fueron trasladados hacia los frutos en maduración para asegurar un mayor contenido de azúcares (Winkler 1980; Catania y Avagnina 2007). Investigaciones realizadas por Almanza et al. (2012) explican que bajo condiciones tropicales la planta no detiene el crecimiento, como ocurre en climas templados y la fotosíntesis continúa.

Blouin y Gumberteau (2004); García y Xirau (2011), explican la importancia de los SST en la elaboración de vinos de calidad; esta consiste en que los azúcares acumulados en los frutos de la vid son transformados en etanol, $\mathrm{CO}_{2}$ y otros compuestos químicos por un proceso de fermentación alcohólica. Por tanto, González (2003) afirma que la calidad óptima de un vino se obtiene con racimos en los que el contenido en azúcares es adecuado al grado alcohólico que se requiere y no precisa corrección enológica; por tanto Almanza et al. (2012) sugiere que el contenido de sólidos solubles totales, en uvas para elaboración de vinos, debe estar cercano a los $23^{\circ}$ Brix, coincidiendo con los valores reportados en esta investigación.

\section{Acidez Total Titulable}

La ATT presentó una disminución a través del tiempo con diferencias significativas desde los 143 ddp a los 173 ddp, sin embargo, con la poda mixta se tuvo la mayor ATT desde los 158 ddp con 13,16 $\mathrm{g} \mathrm{L}^{-1}$ de acido tartárico (Figura 2). Durante la etapa de envero, a los 143 ddp la poda larga mostró los valores más bajos de ATT con 18,9 $\mathrm{g} \mathrm{L}^{-1}$ condición que se mantuvo hasta el momento de la vendimia con $6,88 \mathrm{~g} \mathrm{~L}^{-1}$. Estos valores están dentro del rango reportado en los trabajos de Catania y Avagnina (2007) quienes afirman que con el avance de la madurez, la acidez rápidamente

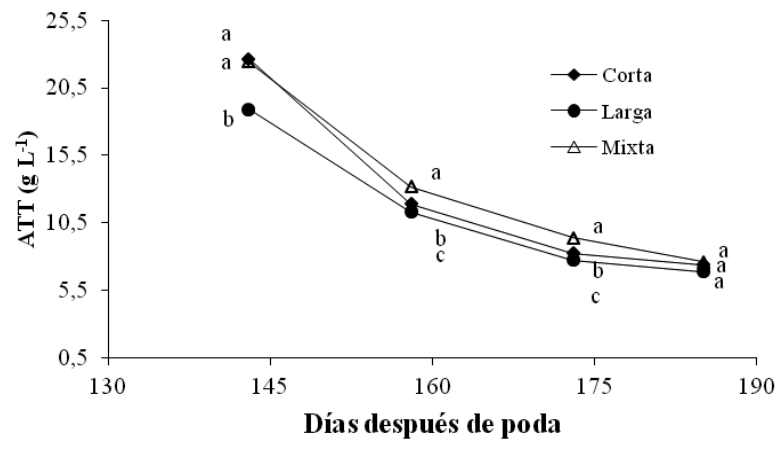

Figura 2. Comportamiento de la Acidez Total Titulable (ATT) durante la maduración de las bayas de 'Sauvignon Blanc' bajo diferentes tipos de poda. ns: no hay diferencias significativas.

baja hasta llegar a 6-7 $\mathrm{g} \mathrm{L}^{-1}$ expresados en ácido tartárico, cantidad compatible con los aspectos gustativos del vino. Dokoozlian (2000), menciona que desde la etapa herbácea hasta el final del envero, la acidez total, representada por los ácidos orgánicos (tartárico y málico), aumentan gradualmente a medida que evolucionaba y crece la baya, encontrándose que los niveles más altos se presentan durante el envero y luego se reducen a través del período de maduración. Por tanto, la disminución de la ATT de los frutos en plantas con podas largas, puede deberse según Aleixandre (2006) y Agustí (2008) a que los acidos tartárico y málico son degregados por fenomenos de dilución, provocada por acumulación de agua, por el consumo durante el proceso respiratorio (Kays 1997) o por que el ácido málico, se transforma en azúcar hacia el final de la maduracion.

La poda mixta presentó el valor más alto de ATT con 7,63 $\mathrm{g} \mathrm{L}^{-1}$, durante la vendimia seguido por el testigo (poda corta), con 7,40 $\mathrm{g} \mathrm{L}^{-1}$. Estos valores difieren de los contenidos de ácido tartárico, encontrados en las variedades Cabernet Sauvignon y Shiraz en 
las investigaciones de Pérez (2003), quien menciona que para estas variedades la ATT, se deben situar entre los 4,5 y los 7,0 $\mathrm{g} \mathrm{L}^{-1}$, los valores altos encontrados en la investigación, posiblemente se dieron debido a las altas precipitaciones presentadas durante el ensayo, lo cual hizo que se presentara mayor cantidad de follaje generando mayor sombreamiento en los racimos. Al respecto, Freeman (1983), en la observación realizada en la variedad Shiraz, menciona que las plantas presentan mayor desarrollo vegetativo, cuando son sometidas a un exceso de agua o a podas con un alto número de yemas, lo cual retrasa la acumulación de azúcar debido a un incremento en el rendimiento; además la acidez y el pH de las bayas son mayores que en aquellas vides sin suplemento hídrico. Al respecto Reynier (1995), menciona que son tres factores los que determinan la dinámica de la acidez: la temperatura, que disminuye la acidez favoreciendo las combustiones respiratorias; el vigor de la planta, que favorece la producción de ácidos orgánicos durante el período de crecimiento y reduce las posibilidades de degradación durante la maduración y finalmente, el suministro hídrico, que causa una dilución cuando es tardía o que favorece la síntesis durante el período de crecimiento.

\section{Índice de madurez}

Aunque el IMT incrementó con el tiempo, solo hubo diferencias estadísticas a los 143 y 158 ddp, en este punto el mayor IMT se obtuvo con la poda larga con 1,32; seguido por la poda corta (testigo) con 1,24 y la poda mixta con 1,11 siendo el más bajo. En los demás puntos de muestreo no hubo diferencias significativas. Teniendo en cuenta que el IMT es la relación entre los SST/ATT, la poda actúa como un regulador fisiológico del metabolismo de la planta, dependiendo del número de yemas que se proyecten, debido a que la actividad fotosintética es dependiente del área foliar resultante del tipo de poda, al respecto, Zufferey y Murisiert (2006) afirman que para una buena maduración de los frutos es imprescindible el control de la superficie foliar. Además, Martínez de Toda (1991) menciona que de acuerdo con la cantidad y longitud de pulgares y sarmientos dejados en la poda, con las sustancias de reserva acumuladas en estas estructuras se podría contribuir a un control adecuado del IMT; posiblemente esta fue una de las razones, por las cuales con la poda larga se presentaron los mayores valores.

Al momento de la vendimia la poda larga alcanzó el valor más alto con 3,42 seguido por la poda corta con IMT de 3,16 (Figura 3). Blouin y Guimberteau (2004), afirma que este índice sirve como referencia para considerar una cosecha óptima desde el punto de vista vitivinícola, además Jubileu et al. (2010) coinciden en asegurar que los SST y la ATT presentan importancia fundamental en el monitoreo del punto de cosecha de frutos

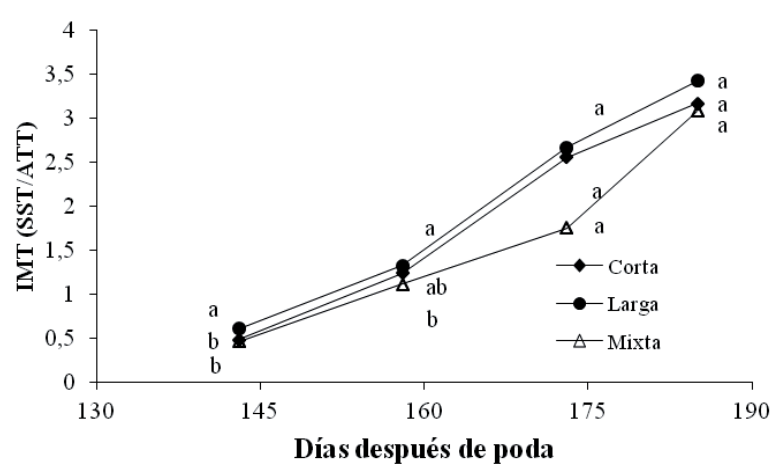

Figura 3. Comportamiento del Índice de Madures Técnica (IMT) durante la maduración de las bayas de 'Sauvignon Blanc' bajo diferentes tipos de poda. ns: no hay diferencias significativas. 
de uva, posibilitando un mejor control de la calidad de la materia prima para elaboración de vinos. Por ser el IMT una medida que cambia perceptiblemente a través del período de maduración de la fruta y que indica las condiciones aproximadas en que debe efectuarse la cosecha (Auda 1977).

Los valores de IMT obtenidos al inicio del proceso de maduración fueron bajos, debido al bajo contenido de azúcar y el contenido alto de ácidos en los frutos. A medida que la uva madura, la proporción de azúcares inicia a aumentar y los ácidos van disminuyendo, por ser parte de la reserva energética de los frutos y que son gastados durante la maduración (Kays 1997). Para la investigación la poda mixta presentó el IMT más bajo con 3,07 debido posiblemente a que tanto los contenidos de SST y la ATT se vieron afectados por la precipitación y la baja radiación solar, presentados durante la investigación por el fenómeno de la niña, lo que afectaría la habilidad fotosintética de las hojas y la carga floemática de sacarosa como proceso que requiere energía (Turgeon y Medville 2004) y de otra parte, por la relación fuente vertedero haciendo que la relación SST/ ATT fuera menor. La importancia del IMT, según Angón et al. (2006), radica en que la proporción entre azúcar y acidez proveen a muchas frutas su sabor característico, además de ser un indicador de la madurez comercial y organoléptica, que permite mayor calidad al producto final.

\section{CONCLUSIONES}

El tipo de poda no tuvo un efecto directo sobre la acumulación de sólidos solubles totales, aunque se evidenció un incremento en forma continua a medida que las bayas ganaban maduración, obteniéndose con la poda larga el mayor contenido $\left(22,76^{\circ}\right.$ Brix), de azúcar. Con la poda larga se obtuvieron los valores óptimos de Sólidos Solubles Totales, Acidez Total Titulable e Índice de Madurez Técnica requeridos para la elaboración de vinos de alta calidad con la variedad Sauvignon Blanc en la región vinícola de Sutamarchán, Boyacá, Colombia.

\section{REFERENCIAS}

Acevedo, C., Ortega, S. y Moreno, Y. 2004. Effect of three levels of water application during post setting and post-veraison over vegetative development, productivity and grape quality on Cv. Cabernet Sauvignon. Acta Hortic 646:143-146.

Agustí, M. 2008. Crecimiento y maduración del fruto. En: Azcon-Bieto, J. y $M$. Talón. (eds): Fundamentos de fisiología vegetal. Segunda edición. McGrawHill Interamericana. España, p656.

Aleixandre, J. 2006. La Cultura Del Vino. Cata Y Degustación. Ed. Universidad. Politécnica. Valencia, p2006-345.

Almanza, P. 2011. Determinación del crecimiento y desarrollo del fruto de vid (Vitis vinífera L.) bajo condiciones de clima frío tropical. Tesis Doctorado Ciencias agropecuarias, área agraria. Universidad Nacional de Colombia. Fisiología vegetal, p145.

Almanza, P., Serrano, P. y Fischer, G. 2012. Manual de viticultura tropical. Universidad Pedagógica y Tecnológica de Colombia, Tunja, p119. 
Angón, P., Santos, N. y Hernández, C. 2006. Índices para la determinación de las condiciones óptimas de maduración de un fruto. Universidad Tecnológica de la Mixteca, Instituto de Agroindustrias. Temas de Ciencia y Tecnología 10(30):3-8.

AOAC. 1990. Offcial methods of analysis. 15th ed. Association of Official Analytical Chemists, Arlington. VA. p128.

Auda, C. 1977. Índices de madurez de frutas. Antecedentes recopilados de investigaciones nacionales y extranjeras. Alimentos 5(3):33-46.

Blouin, J. y Guimberteau, G. 2004. Maduracion y madurez de la uva. Ediciones MundiPrensa, Madrid, p157.

Camacho, M. 2012. Conversación personal. Febrero 2012, viñedo Aikarim.

Catania, C. y Avagnina, S. 2007. Curso superior de degustación de vinos. Inta, Eea Mendoza. INTA. Argentina.

Champagnol, F. 1984. Éléments de physiologie de la vigne et de viticulture générale. Ed Champagnol. Montpellier, p331.

Dokoozlian, N. 2000. Grape berry growth and development. In: Raisin production manual, 30-37. University of California, Agricultural and Natural Resources Publication. Oakland, CA, p3393.

\section{Ferreyra, R., Selles, M., Peralta, J., Burgos, L.} y Valenzuela, L. 1998. Efecto del estrés hídrico aplicado en distintos periodos de desarrollo de la vid cv. Cabernet
Sauvignon en la producción y calidad del vino. Santiago, Chile. AgricTec (Chile) 62:406-417.

Freeman, B.M. 1983. Effect of irrigation and pruning of Shiraz grapevines on subsequent red wine pigment. Am. J. Enol. Vitic 34: 23-26.

García, L. y Bernabé, G. 1997. La viticultura del Jerez. Mundi-Prensa, Madrid, p163.

García, J. y Xirau, M. 2011. Técnicas básicas de análisis en enología. En: http://www. frutales.files.wordpress.com/2011/04/ técnicas.básicas.análisis.enología.html. [2 Junio 2012].

González, M. 2003. Estudio ecofisiológico y agronómico de cuatro sistemas de conducción de la vid (Vitis vinífera L.): cubiertas vegetales simples versus divididas. Producción vegetal. Fitotecnia. ETSIA. Politécnica de Madrid. Madrid, p280.

IDEAM. 2012. Instituto de Hidrología, Meteorología y Estudios Ambientales. Sistema de Información Nacional Ambiental. Estación: 24015300 Villa de Leyva.

Jubileu, B., Sato, J. y Roberto, S. 2010. Caracterizaçao fenológica e produtiva das videiras "Cabernet Sauvignon" e "Alicante" (Vitis vinífera L.) producidas fora de época, no norte do Paraná, Rev. Bras. Frutic. 32(2):451-462.

Kays, S. 1997. Postharvest physiology of perishable plant products. Exon Press, Georgia. 
Lavín, A., Lobato, A., Muñoz, I. y Valenzuela, J. 2003. Viticultura: poda de la vid. Cauquenes, Chile. Instituto de Investigaciones Agropecuarias. Boletín INIA- $N^{0}$ 1. En: http://www.inia.cl [Enero 2012].

Martínez de Toda, F. 1991. Biología de la Vid. Fundamentos biológicos de la viticultura. Mundi-Prensa, Madrid, p346.

Ministerio de Medio Ambiente y Medio Rural y Marino de España (MAPA). 2008. Variedades de vid. En: http://www. mapa.es/app/exposiciones/vid/www/ imagenes/variedades_uvalistado.html [5 Mayo 2012].

Parra, C.A. y Hernández, J.E. 1997. Fisiología, postcosecha de frutas y hortalizas. Bogotá. Universidad Nacional de Colombia. Facultad de Ingeniería, p64.

Pérez, C. 2003. Análisis (químico) y control (digital) en la producción del vino. En: http://redesformacion.jccm.es/aula_ abierta/.../vino.pdf [12 Marzo 2012].

Pszczolkowski, T., y Bordeu, E. 1984. Posibles causas del deterioro de la calidad del vino en parronales y viñedos vigorosos. Rev. Frut. 5(1):23-26.

Reynier, A. 1995. Manual de viticultura. 4a ed. Ediciones Mundi-Prensa, Madrid, España, p382.
Sipiora, M. 1995. Influencia del aclareo manual, el aclareo con etefón y el deshojado sobre la producción y la composición del fruto de Moristel, Cariñena y Chenin Blanc. Vitic/Enol. Prof 45:16-24.

Smart, R. y Robinson, M. 1991. Sunlight into Wine. A handbook for wine-grape canopy management. WinetitlesAdelaide. Am. J. Enol. Vitic 35(3):117-123.

Togores, J. y Fernández-Cano, L. 2011. Tratado de Viticultura general. $4^{\mathrm{a}}$ ed. Ediciones Mundi-Prensa, Madrid, España, p405.

Turgeon, R. y Medville, R. 2004. Phloem loading. A reevaluation of the relationship between plasmodesmatal frequencies and loading strategies. Plant Physiology 136:3795-3803.

Winkler, A. 1980. Desarrollo y composición de frutas En: Viticultura. CECSA. México, p163-202.

Zamboni, M., lacono, F., Fraschini, P. y Parenti, A. 1991. Influenza della carica di gemme e della lunghezza di potatura sulla produttivita e sulla'agostamento del vitigno Croatina. Vignevini 12:51-55.

Zufferey, V. y Murisier, F. 2006. Distance interligne et hauteur de la haie foliaire (II). Revue Suisse de Viticulture, Arboriculture. Horticulture 38(3): 161-164. 